\title{
LA TERRITORIALIDAD DEL INDÍGENA AMERICANO DE LA TIERRA ALTA DEL NORTE DE LA BAJA CALIFORNIA*
}

\author{
Por' \\ Ralph Michelsen**
}

\begin{abstract}
RESUMEN
Ralph Michelsen explora la idea de territorialidad entre los indígenas de Baja California como sujetos de otro tipo de realidad; un ticmpo cuando el usufructo de la ticrra se ceñía a reglas aborígenes particulares en contraste con las formas de tenencia de la tierra actuales y la noción de "país" que en el mundo prevalece. Su finalidad se logra mediante una reconstrucción histórica que le permite hacer propuestas sobre la percepción del espacio geográfico por los miembros de unidades lingǘísticas y/o de parentesco.
\end{abstract}

\begin{abstract}
Ralph Michelsen explores the idea of territoriality among the native inhabitans of Baja California as subjects of another type of reality; a time when the use of land was in accordance with particular aboriginal rules in contrast with the present forms of land ownership and the notion of "country" that prevails in the world. His objective is reached on the basis of a historical reconstruction that allows him to forward proposals on the perceptions that the members of linguistic or kinship units have of the geographical space.
\end{abstract}

Al ver un mapa del norte de la Baja California, que fuera elaborado con el propósito de delimitar los territorios de los diversos grupos indígenas americanos, uno se encuentra con territorios catalogados como: tipai, paipai, kiliwa, nakipa, y así sucesivamente. Estas delimitaciones generalmente se refieren a algún tipo de separación de los diferentes grupos, ya sea cultural o políticamente. Desde este punto de vista, la territorialidad tiene sentido para nosotros en términos conceptuales, probablemente porque una de las maneras por medio de las cuales designamos nuestras propias divisiones culturales y políticas es trazando líneas en un mapa. A no ser que específicamente se señale que las líneas fronterizas designan zonas económicas, zonas geológicas, regiones lingúísticas, o algo por el estilo, generalmente aceptamos las fronteras como divisiones políticas.

* Traducido del inglés por Carlos Cortez.

**.Antropólogo, Facultad de Ciencias Sociales, Universidad de California en Irvine. 
Hasta tiempos muy recientes, los antropólogos han aceptado tales interpretaciones culturales como expresiones útiles de la realidad. Sin embargo, con la introducción del concepto Emic-Etic de Pike (1984) y con el desarrollo de la antropología del conocimiento, hemos iniciado la búsqueda de otro tipo de realidad. $\iota$ Cómo perciben los sujetos mismos su mundo en sus múltiples aspectos, incluso la idea de territorialidad? ¿Aceptaría, por ejemplo, un indio paipai, que las fronteras de nuestros mapas están en concordancia con la visión del mundo que tiene su tribu? ¿Estarán de acuerdo los kiliwas con nosotros acerca de los derechos a, o la exclusión de, los territorios que nosotros hemos asignado a los paipais o a los nakipa?

Hoy en día la respuesta a estas preguntas es, en general, afirmativa, puesto que la mayoría de los indígenas viven en territorios designados por el gobierno mexicano como ejidos o territorios de indígenas. Sin embargo, si miramos al tan llamado mapa tribal de los territorios aborígenes, la respuesta debería ser negativa. Este documento se basa en la reconstrucción histórica reciente, y el propósito es tratar de clarificar el concepto de "territorialidad" desde el punto de vista de los indígenas, y ofrecer algunas observaciones concomitantes que están relacionadas con la percepción indígena del espacio geográfico.

La búsqueda de la concepción de territorialidad de un grupo de sujetos que ya no existen, excepto como historia, puede parecer normal; pero, a partir de que nosotros hemos delineado nuestras concepciones acerca de los antiguos territorios de los nativos, puede ser útil compararlas con aquéllas de la gente que se estudia.

Esto no presenta grandes dificultades, porque los miembros más viejos de un grupo por lo común recuerdan los territorios específicos y bien definidos, donde en el pasado su banda mantenía derechos exclusivos de residencia sobre determinadas lagunas o charcas. Algunas regiones donde recolectaban sus comestibles eran de posesión exclusiva de la banda, en tanto que otras regiones de recolecta eran explotadas conjuntamente por varias bandas, especialmente en las playas y en las montañas más altas, donde se recolectaban piffones y bellotas. También ciertas tribus poseían derechos exclusivos sobre las cimas de determinadas montañas y hay un caso particular de una isla en el golfo de California donde el espíritu de sus shamanes residía después de su muerte.

Para entender mejor el significado de la territorialidad en los indígenas, es necesario dar una breve explicación de la organización social y de las normas de subsistencia que tenían. Exceptuando a los semihorticultores cucapás, el patrón de subsistencia de los indios del norte de la Baja California era predominantemente el de cazadores y recolectores; se practicaba la pesca de peces y mariscos, pero con muy poca tecnología 
marítima. El tamaño de las unidades poblacionales generalmente no excedía de cincuenta personas, pero por lo común el número era de veinte a veinticinco individuos (Owen y Michelsen, $\mathrm{s} / \mathrm{f}$ ). Hay datos referentes al hecho de que en el tiempo en que Arrillaga tuvo el primer encuentro con estos indígenas, muchas de las bandas constaban de cuatro a ocho chozas, lo que podría indicar una población de quince a treinta individuos (Owen y Michelsen, s/f). Debido a las variaciones de las estaciones que afectaban las existencias de comestibles disponibles, las bandas de indígenas se mudaban a diversas regiones donde obtenían diferentes comestibles, por ejemplo, a las montañas a recolectar bellotas y pinones en las postrimerías del verano y en el otoño, y a las playas en el invierno cuando otras fuentes de víveres escaseaban o no existían. Por lo tanto, tenemos una población formada por pequefias bandas que estaban en constante movimiento para explotar diversos recursos según la estación, pero que tenía posesión exclusiva de un sitio que era su morada-base. En la mayoría de los casos, la morada-base era ocupada por una sola familia y sus parientes. Todas estas moradas-base se encontraban a menos de 3,500 pies de altitud y por lo general a una distancia considerable de las costas del golfo de California y del océano Pacífico.

La organización social era un reflejo de los patrones de subsistencia, de naturaleza predominantemente familiar aunque era a la vez lo suficientemente flexible para permitir cierto grado de libertad en las relaciones externas. La unidad social ampliada era (y es) el chumul. ${ }^{1}$ Los miembros del grupo son cooperativos con los miembros de su grupo pero no se pueden casar entre sí. En el pasado, los chumules y sus respectivas subdivisiones eran unidades de morada-base, asociadas con regiones geográficas específicas. Actualmente, como los indios se han unido en varios ejidos indígenas, los chumules han perdido todo excepto el recuerdo de su regionalidad (Owen y Michelsen, s/f). No obstante, la comprensión de la naturaleza del chumul es básica para entender la prehistoria de la Baja California, aunque en los tiempos modernos esta unidad social ha perdido mucho de su significado (Owen y Michelsen, s/f).

La patrilocalidad se tenía por ideal y por lo general se asumía. Las reglas de la exogamia eran (y son) bilaterales y extremas entre los paipai (Owen, 1963). No se permitía el matrimonio entre los miembros del mismo chumul (o ichiupu) o con los parientes maternos o con los miembros del chumul

1 N. del E. Chumul se utiliza para designar a aquellas unidades de individuos que reconocen su descendencia a través de la línea patema. Es un término originario de las lenguas yumanas. A través del tiempo distintos investigadores lo identifican y le dan ciertas escrituras como son: Chumul, shumul, shamul, cimul, shimul. 
materno (Esta última prohibición tal vez no se observaba entre los tipai o kumiai). Obviamente, en una población de baja densidad — probablemente menos de un habitante por milla cuadrada- y con estrictas reglas de exogamia, era necesario ir lejos para hallar un consorte aceptable.

Primitivamente, la lucha por la subsistencia requería la cooperación de los miembros de la familia nuclear principalmente, y en menor grado de sus parientes. El chumul, en tanto banda patrilocal o unidades, era la extensión de la unidad económica excepto en estaciones de cosechas abundantes. Era necesaria una cooperación más extensa para eventos ceremoniales, raras veces, para actividades militares. La patrilocalidad tendía a mantener la identidad de un chumul con determinadas regiones geográficas. Los mismos patrones pueden percibirse en la actualidad en Santa Catarina.

Paradójicamente, conforme los hombres de una familia morían o desertaban de su familia, se desarrolló la matrilocalidad. Los hijos permanecían con su madre; sin embargo, como el sistema chumul seguía la línea masculina, se mantenía esta localidad chumul. Actualmente, las hijas a menudo engendran hijos con forasteros - simples transeúntes y generalmente extranjeros- sin afiliación con un chumul. En tales casos, y hay muchos, los descendientes de tales uniones reclaman afiliación con el chumul materno.

Algunos chumules se subdividieron y ocuparon muchas diferentes moradas-base abarcando regiones que les fueron concedidas en exclusividad por otros chumules. Un gran chumul, kwatl, tenía grandes subdivisiones que cruzaban las fronteras lingüísticas. Existían dos linajes Kwatl entre los cucapá, uno de ellos reconocido como de origen diegueño (Kelley, 1942:680). Kwatl, o kwatl kumiai, es un chumul diegueño grande.

Entre los paipai había al menos tres divisiones kwatl importantes, y una de ellas estaba dividida en tres ramas menores, para diferenciarlas; al nombre se le añadía un sufijo que indicaba la localidad o alguna otra característica. Así tenemos una subdivisión kulwat que hablaba paipai, conocida como Kulwat wi pak, que ocupaba la parte baja del desierto en el lado oriental del extremo sur de la sierra de Juárez (se afirma que kul es apócope de kwatl; la traducción de kul es liebre, la traducción de kwatl es piel o cuero, y la traducción de wat es rojo). Esta rama ocupaba los cañones al norte de Arroyo Grande que tenían abundante agua. La subdivisión Kulwat chi sil vivía al oeste de Portezuelo de Jamau, mientras que la rama Kulwat wat ja puti vivía más al poniente en el llano del Álamo. Al norte de Kulwat wi pak se encontraba la rama miakwa que hablaba paipai, y que tenía su morada base en el cañón de La Parra. En la actualidad, la subdivisión el Miakwa vive en Santa Catarina, y tiene lazos tradicionales con la rama cucapá del río Hardy. 
Al sur de donde habitaba la rama Kulwat wi pak de Arroyo Grande vivió la subdivisión Chuwilubitai pakawa que habla kiliwa. Alrededor de 1840 se mudaron a las faldas de baja altitud de la parte norte de la sierra de San Pedro Mártir. La mayoría de la rama Chuwilubitai pakawa todavía vive allí, integrada — aunque no muy amistosamente - con la rama Japolkelawa de habla kiliwa.

E1 ya extinto chumul Juigrepa (probablemente no sea una autodenominación ya que se traduce como "bocas duras") ocuparon los cañones de palmas al sur del Japolkelkawa. Meigs supo, por unos informantes kiliwas, que el chumul Juigrepa hablaba una lengua muy diferente de la kiliwa y que el chumul kiliwa lo veía como enemigo (1939:84). Dos nombres de bandas juigrepas fueron obtenidos de informantes paipai y kiliwa: la $K o^{\prime}$ sil localizada en Cerro Borrego y Pozo Salado, al oriente del desfiladero de San Matías; y la Xa' wi yakipa que se encontraba en los alrededores de Pozo Salado y del Cañón Esperanza. Es muy probable que los kiliwa exterminaran estas bandas en disputas sobre el pozo de agua salobre de San Felipe.

Los antepasados de ambas bandas, japolkelkawa y Chuwilubitai, pescaban en San Felipe. Los informantes kiliwa de Meigs afirmaron que el linaje Xwatch ocupó el extremo superior del Cañón Carrizo en el lado norte del valle Trinidad (1939:18). Los informantes paipai afirman que ése era territorio kwatl kweipei. Parece ser que el linaje Kwatl kweipei compartía localidades con varios otros linajes que incluían el Xamsulch, el Qshaqsh, y el Kwatl wirr.

El $X a^{\prime}$ sa' er ocupaba el extremo inferior de El Carrizo y también el extremo oeste del valle Trinidad. El desagüe costero lo ocupaban todos los linajes arriba mencionados. El Kwatl wirr parece ser que reclamaba $\mathrm{La}$ Grulla y Santo Tomás como su territorio, y también abarcaba partes de las bandas mixtas situadas río arriba de la misión en San Vicente.

La banda Qshaqsh se ubica en San Vicente así como en Rincón y en La Poza, una zona muy extensa para ser ocupada por una sola banda. Ellos afirman haber recolectado agave en el desierto oriental y haber ido hasta el Pacífico por pescado; su ruta sigue el río San Vicente, en línea casi directa desde la costa del Pacífico, cerca de Punta San Isidro, hasta un camino bien conocido que va de Rincón al desierto. La banda Xamsulch vivía cerca de San Vicente, mientras que otras bandas Xamsulch ocupaban el arroyo San Rafael desde el pie de la sierra de San Pedro Mártir hasta la playa. Un afluente del mismo arroyo, el arroyo Seco, también estaba ocupado por la banda Xamsulch - y lo compartía con el Kwatl kweipei y el Xwatch.

El Arroyo Los Coches lo compartían los Kwatl wirr y Kwatl kweipei. Los $K$ watl wirr de habla paipai eran los que vivían más al norte, lo que indica que la ocupación paipai llegaba hasta Punta Banda. Tierra adentro 
sobre la cordillera costera en Agua Herbidora, al noroeste de El Álamo, vivian los Qui noh quienes estaban, desde el punto de vista lingüistico, más estrechamente relacionados con los tipai. Al sur de los xamsulch, situados alrededor de San Telmo, se encontraban los llamados el xau ("trituradores de mezcal"), que parece ser un término despectivo. Son de identidad dudosa, los paipai afirman que los yel xau hablaban kiliwa, pero los kiliwa a su vez afirman que hablaban una lengua diferente a la kiliwa. Tales aseveraciones de los indios, a menudo las hacen aunque sólo difieran unas cuantas palabras o si el acento es diferente al de ellos.

Los indios que vivían al suroeste de los kiliwas eran llamados nakipa ("gente de occidente" o "de donde se pone el sol"). Este mismo grupo era llamado Yak ka kwal ("cosa cruda") por los paipai. Existe cierta confusión respecto a los nombres de las bandas o chumules, debido a las diferentes denominaciones dadas por informantes de diferentelengua. Los paipai $x$ watch son llamados kekwalp por los japolkelkawa de habla kiliwa. Xa'sa'er, una denominación paipai, es igual que jasigre, en kiliwa (Meigs, 1939:18).

A pesar de que en los testimonios hay confusiones y contradicciones, parece existir una evidencia clara de que la gente de varios chumules, y de bandas que pertenecían a varios chumules, en la mayoría de los casos tenían una idea clara del territorio que pertenecía a su gente. Por lo menos algunos de los chumules poseían territorio en exclusividad. Otros chumules compartían territorios con sus aliados. Hay que observar que los territorios compartidos se agrupaban cerca de la Misión San Vicente. En la actualidad no existen comunidades indígenas cercanas a los lugares donde estaban las misiones costeras. Algunos de los habitantes de Santa Catarina - los qshaqsh, los xamsulch, y los kwatl kweipei- afirman que su gente vivió antiguamente más cerca de la costa en un mismo período. Tienen vivos recuerdos del terreno en Los Dolores, que fue destruido por inundaciones antes de la primera guerra mundial. Es probable que al menos parte de la estructura de las bandas mixtas se deba a la misionización; indudablemente ese es el caso hoy día en Santa Catarina. La población actual de Santa Catarina incluye afiliaciones de la mayoría de los chumules mencionados; sin embargo, el amalgamiento de las bandas y de los chumules es más aparente que real, como se señalará en la conclusión.

Los kiliwas afirman que, tradicionalmente, los territorios de la banda eran exclusivos a tal grado que se tomaban medidas hostiles contra los intrusos. En esos casos, los intrusos eran aquéllos con quienes no existían lazos, ni de consanguinidad ni de afinidad.

Los paipai y los ko'al de Santa Catarina se protegen celosamente contra la intromisión de los forasteros en su ejido, aunque éstos sean indígenas. Los forasteros que se casan con un miembro del ejido son tratados como 
forasteros toda su vida - no sólo porque viene de lejos, sino también porque la única bienvenida que se puede recibir es la de un pariente $u$ otro miembro del mismo chumul, pero principalmente por lo primero-. Los demás son extranjeros, a no ser que se pueda comprobar algún tipo de ancestro o linaje en común. Un indígena kiliwa de arroyo León, ex marido de una india paipai, recientemente solicitó residencia en Santa Catarina alegando que sus hijos tenían derechos de residencia por derechos maternos; su solicitud fue rechazada. Hay varios chuwilubitai (kiliwa) que viven en Santa Catarina, pero todos ellos son hijos de madre paipai. Los kiliwa no son muy bienvenidos y todos excepto un individuo, que tiene fuertes lazos familiares, viven a dos o tres millas del pueblo.

Las relaciones entre chumules de grupos linguísticos similares son variadas. Los mikawa son hostiles hacia los kulwat, pero los kulwat son aliados de los chuwilubitai. Los kulwat son hostiles con los chumules de habla paipai que antiguamente ocuparon los cañones de la cordillera costera. Los japolkelkawa son más cordiales con los kwatl kweipei que con los chuwilubitai, y así sucesivamente. La externalización de esto se refleja en abierta hostilidad, como ocurrió durante el movimiento magonista de 1911 en el norte de la Baja California (Owen, 1963).

Los territorios actuales de las bandas y los chumules amalgamados que sobreviven, están siendo establecidos formalmente con la ayuda y el estímulo de las agencias gubernamentales. Los indígenas consideran los territorios asignados como reservaciones para el uso exclusivo de ciertas familias que han ocupado las tierras por varias generaciones. Los vecinos que no son indígenas a menudo consideran las tierras como si fueran más bien ejidos y no necesariamente como tierras asignadas permanente y exclusivamente para los indígenas. Sin embargo, en la actualidad la cuestión es inconsecuente, porque la población indígena es muy estable en casi todas partes y sus vecinos mexicanos respetan sus derechos. Las fronteras legales se han trazado conforme a las normas legales y culturales de la sociedad dominante y están de acuerdo con los valores contemporáneos de los indígenas hasta donde éstos son entendidos. La mayoría de los indígenas están bien integrados a la economía del peso y en muchos casos su posición económica es igual que la de sus vecinos que no son indígenas. Las condiciones van mejorando aún más gracias a los programas gubernamentales de desarrollo económico.

Como ya se ha dicho anteriormente, la mezcla de varios chumules en Santa Catarina es más aparente que real. De hecho, la reservación es probablemente un microcosmos de antiguos y más extensos territorios. Cada chumul o banda que vive actualmente en la reservación de Santa Catarina tiene su propia base delimitada. Los afiliados kulwates y ko'al 
tienen derechos exclusivos en El Rincón. Un indígena ko'al con su esposa kiliwa (Xapi pakawa) ocupan el manantial en Agua Escondida. Una familia kulwat ocupa el manantial en Agua Colorada, y algunos de sus parientes kiliwas (chuwilubitai) viven en las cercanias. En la región central, alrededor de sitio de la misión, la población es más densa debido a la mayor abundancia de agua y tierras irrigables. El agua es compartida tanto para el uso doméstico como para irrigar los cultivos. El pueblecito lo forman unas casas dispersas, o recintos, que reflejan la naturaleza, centrada en la familia, de su organización social. Hay una agrupación de kawatl kumeyai cercana a la entrada de la comunidad. Este chumul es de reciente fundación, ya que un cantante kwatl kumeyai se casó con una mujer paipai (Owen, Walstrom y Michelsen, 1969:102). A unos cuantos cientos de metros al oriente se encuentra una agrupación de ko'al. Al otro lado del valle vive una familia miakwa; más lejos al otro lado del valle, hay una unidad de parientes solteros de edad madura que son de afiliación de chumules mixtos. Un afiliado xamsulch vive al otro lado del arroyo donde viven unos miakwa, y así por el estilo. La distribución espacial está disenada convenientemente para que las personas puedan pasar por alto la presencia de los otros. La distancia es tal que nadie se siente obligado a ser sociable con los vecinos y, ciertamente, algunas mujeres que no tienen parientes pueden estar meses sin saludar a otras mujeres que viven a poca distancia de ellas.

Un sociograma de la comunidad revela que las normas de visitas se basan principalmente en lazos de consanguinidad. Como es de esperarse, la familia nuclear es la unidad económica básica, excepto en casos de extrema necesidad; los parientes no son una parte importante de la unidad económica a no ser que ellos vivan en la misma casa. Se supone que los individuos son autosuficientes. La cooperación fuera de la familia ni se requiere ni se espera, lo que ocasiona disgustos tanto a los misioneros como a los dirigentes de proyectos de desarrollo de la comunidad. Las consecuencias de los rasgos y actitudes antes mencionadas se manifiestan en actividades sociales tales como bailes y reuniones en los que se prepara barbacoa. Cada individuo debe ser invitado personalmente a cualquier evento o de lo contrario no asistirá. Una invitación general no es suficiente para motivar a una familia a introducirse en la casa de otra familia sin que previamente la familia invitada haya sido persuadida personalmente de que será bienvenida.

En conclusión, la territorialidad parece estar ligada a la ocupación y explotación por unidades familiares que eran subdivisiones de chumules. Estos eran verdaderamente identificados como poseedores de territorios definidos, algunos, sin duda, compartidos con otros chumules que eran sus 
aliados y con los cuales mantenían un intercambio de consortes elegibles. Los depósitos de agua que eran ocupados como morada-base no se compartían excepto para eventos ceremoniales, en el interior. Más cerca de la costa, especialmente alrededor de San Vicente, posiblemente hubo una banda mixta más grande; pero aún nos queda el problema de evaluar los efectos de las misiones. Hay una posibilidad de peso acerca de que existía una amalgama de bandas alrededor de lugares con agua y tierras que podían mantener las nuevas normas de subsistencia basadas en la horticultura. La mayoría fue excluida de los sitios donde estaban las misiones costeras debido a la intromisión de extranjeros.

Parece ser que las regiones más alejadas de la actividad de los sitios de las misiones fueron poseídas en exclusiva por bandas que eran miembros de un chumul que poseía territorios bastante grandes. Esto fue verdaderamente cierto en el caso de los kulwat y sus tres subdivisiones. Los miakwa no compartían su territorio con nadie. Los ko'al de habla diegueña ocuparon el territorio inmediato al norte de Santa Catarina. Aparentemente, los kwatl wirr fueron el grupo que tenía en exclusiva La Grulla. A propósito, esto coloca los límites para los paipai en nuestros mapas étnicos convencionales considerablemente más al norte a lo largo de la costa. Los de habla kiliwa son singularmente interesantes, ya que los chuwilubitai estaban (y están) notablemente más ligados a los kulwat que a los japolkelkawa de habla kiliwa.

Si un indígena paipai fuera a dibujar un mapa del territorio paipai, problablemente dibujaría un mapa de la región en que viviría la gente con quien él podría conversar. Sin embargo, si se le pidiera que dibujara un mapa de "su" territorio, lo más probable es que dibujaría un mapa de los lugares a donde él pudiera ir sin peligro. El mapa no se ajustaría ni con un mapa de la región donde se hable su lengua, ni con un mapa dibujado por otro paipai de diferente linaje. Algunos de los mapas dibujados por tales individuos incluirían territorios de los kiliwas o de los cucapás, o de los diegueños. Es decir, para poder dibujar una representación realista del territorio paipai habría que dibujar un sociograma y no un mapa étnico.

\section{BIBLIOGRAFÍA}

KELLEY, William H. 1942. "Cucapá Gentes”. American Anthropologist. No. 44.

MEIGS, Peveril, (ed.) 1939. The Kiliwa Indians of Lower California. Berkeley: Universidad of California Press.

OWEN, Roger C. 1939. "Indians and the Revolution: The 1911 Invasion of Baja California". Ethnohistory No. 10. 
- 1963. "The Social Demography of Baja California". American Anthropological Society, 62d Meeting.

OWEN, Roger C., y Michelsen, Ralph C. s/p, (mimeo).

OWEN, Roger C., Walstrom, Nancy, y Michelsen, Ralph C. 1969. "Musical Culture and Ethnic Solidarity". Folklore No. 82.

PIKE, Kenneth. 1954. Unified Theory of Structure of Human Behavior. Vol.1. Glendale: Summer Institute of Linguistics. 